\title{
Tratamento da disforia pré-menstrual com antidepressivos: revisão dos ensaios clínicos controlados
}

\author{
Treatment of premenstrual dysphoria with antidepressants: review of controlled \\ clinical trials
}

Elie Cheniaux

\begin{abstract}
Resumo
Introdução: Adisforia pré-menstrual (DPM), que acomete entre $3 \%$ e $8 \%$ das mulheres em idade fértil, representa uma forma mais grave de síndrome prémenstrual, na qual predominam as alterações do humor e do comportamento. Acredita-se que haja algum tipo de ligação entre a DPM e os transtornos do humor. Objetivo: Estudar a eficácia dos antidepressivos na DPM. Métodos: Foi realizada uma revisão dos ensaios clínicos controlados com antidepressivos no tratamento da DPM, utilizando-se as seguintes bases de dados: Medline, Literatura Latino-Americana e do Caribe em Ciências da Saúde (LILACS), psycINFO e Biblioteca Cochrane. Resultados: A clomipramina, a fluoxetina, a sertralina, a paroxetina e a venlafaxina se mostraram superiores ao placebo em diversos estudos. Discussão: As mulheres que sofrem de DPM possivelmente apresentam uma disfunção serotoninérgica. Conclusão: Alguns antidepressivos, especialmente os inibidores seletivos da recaptação da serotonina (ISRS), parecem ser eficazes no tratamento da DPM.

Palavras-chave: síndrome pré-menstrual, tratamento, antidepressivos, revisão.
\end{abstract}

\begin{abstract}
Introduction: Premenstrual dysphoria (PMD), which affects between $3 \%$ and $8 \%$ of women during reproductive years, represents a more severe type of premenstrual syndrome. Mood and behavior changes are predominant in PMD. It is believed that there is some kind of link between PMS and mood disorders. Objective: Studying the efficacy of antidepressants in PMD. Methods: We elaborated a review of controlled clinical trials with antidepressants in the treatment of PMD, using the following databases: Medline, LILACS, psycINFO and Cochrane Library. Results: Clomipramine, fluoxetine, sertraline, paroxetine and venlafaxine were superior to placebo in various studies. Discussion: Women with PMD possibly present a serotonergic dysfunction. Conclusion: Some antidepressants, specialy SSRIs, seem to be effective in the treatment of PMD.

Key words: premenstrual syndrome, treatment, antidepressants,
\end{abstract} review.

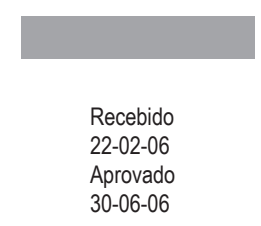

Faculdade de Ciências Médicas da Universidade do Estado do Rio de Janeiro (FCM/UERJ); Instituto de Psiquiatria da Universidade Federal do Rio de Janeiro (IPUB/UFRJ); Coordenação dos Programas de Pós-Graduação de Engenharia (COPPE) da UFRJ (Cheniaux E)

Correspondência para: Elie Cheniaux

R. Santa Clara, 50/1213 - Copacabana - 22041-010 - Rio de Janeiro-RJ - Telefax: (21) 2547-0670

e-mail: echeniaux@globo.com 


\section{Introdução}

A síndrome pré-menstrual (SPM) constitui um distúrbio altamente prevalente entre as mulheres em idade fértil. Em estudos brasileiros, foram encontradas taxas de 35\% (Diegoli et al., 1994) e de 43,3\% (Nogueira e Silva, 2000) entre pacientes ambulatoriais, e de $25,2 \%$ num estudo populacional recente (Silva et al., 2006). Entre os sinais e sintomas mais comuns estão irritabilidade, ganho de peso, edema (principalmente de abdome e mamas), mastalgia, tristeza, fadiga, cefaléia, labilidade afetiva, episódios de choro, ansiedade, tensão e alterações do apetite (particularmente avidez por alimentos ricos em carboidratos, como chocolate). A presença de uma única manifestação clínica durante a fase lútea do ciclo menstrual é suficiente para a caracterização de um quadro de SPM (Ling, 2000). Sua etiopatogenia é desconhecida (Steiner e Pearlstein, 2000).

$\mathrm{Na}$ década de 1980, a SPM foi pela primeira vez incorporada à nosografia psiquiátrica, tendo sido incluída na terceira edição revisada do sistema classificatório da Associação Psiquiátrica Americana, o Manual Diagnóstico e Estatístico de Transtornos Mentais (DSM-III-R), sob a designação de transtorno disfórico da fase lútea tardia (TDFLT), depois rebatizada como transtorno disfórico pré-menstrual (TDPM) na quarta edição, o DSM-IV (Endicott, 2000). A disforia prémenstrual (DPM), conceito que engloba tanto o TDFLT como o TDPM - já que os critérios diagnósticos para ambos são praticamente idênticos nas duas versões da classificação americana -, acomete entre $3 \%$ e $8 \%$ das mulheres durante seus anos reprodutivos, segundo estimativas internacionais. Ela representa um subtipo de SPM no qual as manifestações são mais graves e mais numerosas e em que predominam as alterações do humor e do comportamento. Para a formulação de um diagnóstico de DPM é necessário que ocorra pela menos uma alteração do humor - irritabilidade, tristeza, ansiedade ou labilidade afetiva -, num total de, no mínimo, cinco sintomas; que o quadro clínico cause um grave prejuízo nas atividades ocupacionais, conflitos nos relacionamentos interpessoais ou isolamento social; e que a maioria dos ciclos dos últimos 12 meses tenha sido sintomática. Além disso, esse diagnóstico precisa ser confirmado prospectivamente por pelo menos dois ciclos (Endicott, 2000).

Inicialmente diversas opções terapêuticas, como progesterona, vitamina $B_{6}$, diuréticos, contraceptivos orais, etc., pareciam eficazes na SPM ou na DPM. Todavia a observação de bons resultados se limitava a estudos abertos e não se repetiu quando se realizaram ensaios clínicos com metodologia mais sofisticada (Pearlstein e Steiner, 2000).

Nas últimas décadas, vários psicotrópicos, especialmente antidepressivos, têm sido testados na DPM, o que se apóia num conjunto de evidências clínicas e epidemiológicas que indicam a existência de uma relação bastante próxima entre os transtornos do humor, particularmente a depressão, e a DPM. A depressão é mais prevalente no sexo feminino. Na mulher, os episódios depressivos são mais comuns durante os anos reprodutivos e muito freqüentemente se iniciam ou se tornam mais intensos em períodos pré-menstruais. Além disso, há muitos sintomas em comum entre a DPM e a depressão, sendo bastante alta a taxa de co-morbidade entre esses dois transtornos mentais (Yonkers, 1997). Por outro lado, sugere-se que, como na depressão, diversas alterações clínicas observadas na DPM, como a avidez pela ingestão de carboidratos, estejam relacionadas com uma hipofunção serotoninérgica. De fato, há ensaios clínicos que demonstram que uma dieta rica em carboidratos, essenciais para a síntese do triptofano (precursor da serotonina) no organismo, leva à redução dos sintomas na DPM (Wurtman et al., 1989). Além disso, outros estudos apontam uma boa resposta da DPM ao uso do L-triptofano e da buspirona, medicamentos que afetam a atividade serotoninérgica (Pearlstein e Steiner, 2000). Com relação a exames laboratoriais, foram encontradas algumas anormalidades no sistema nervoso central (SNC) em mulheres com DPM, envolvendo especialmente o sistema serotoninérgico. Elas estão relacionadas, por exemplo, à recaptação plaquetária da serotonina, à sensibilidade dos receptores $5-\mathrm{HT}_{1 \mathrm{~A}}$, etc. (Steiner e Pearlstein, 2000).

\section{Métodos}

Foi realizada uma revisão sobre o tratamento da DPM com antidepressivos. Pesquisou-se todo o conteúdo das bases de dados Medline, Literatura Latino-Americana e do Caribe em Ciências da Saúde (LILACS), psycINFO e Biblioteca Cochrane até 0 ano de 2005. Utilizaram-se os seguintes termos de busca: premenstrual syndrome, premenstrual dysphoria, premenstrual dysphoric disorder e late luteal phase dysphoric disorder. Não se utilizou nenhum termo de busca relacionado a antidepressivos. Na busca foram empregados os seguintes filtros: clinical trial no Medline, ensaio clínico na LILACS, treatment outcome clinical trial no psycINFO e ensaios controlados na Biblioteca Cochrane.

Seguindo os procedimentos anteriores, os resumos de todas as referências obtidas foram avaliados, um a um, somente por este autor. Foram selecionados apenas os ensaios clínicos controlados que avaliaram a eficácia de antidepressivos na DPM, aleatorizados (randomizados) ou não, nos quais a comparação tivesse sido feita com placebo ou com outra substância ativa. Só foram incluídos os estudos cujas amostras eram constituídas unicamente por pacientes que preenchiam os critérios do DSM-III-R para o diagnóstico de TDPLT ou os do DSM-IV para o de TDPM. Não se estipulou número mínimo de pacientes para a amostra estudada. A avaliação da qualidade dos estudos primários não foi considerada na seleção. Não foram incluídos artigos de revisão ou metanálise, cartas, editoriais, resumos em anais de congressos científicos, ou ensaios clínicos abertos. Não houve busca por trabalhos não-publicados. De forma complementar, os artigos selecionados também serviram como fontes de novas referências. Foram contactados, via correio eletrônico, dois autores, Mikael Landen e Cláudio Novaes Soares, para a obtenção de informações sobre artigos que estavam prestes a ser publicados.

\section{Resultados}

A busca inicial nas bases de dados, antes da análise dos resumos, produziu os seguintes resultados: 394 referências foram obtidas no Medline, nenhuma na LILACS, 42 no psycINFO 
e 454 na Biblioteca Cochrane. Numa primeira avaliação dos resumos, 33 referências foram selecionadas. Os motivos mais comuns de exclusão em relação à metodologia dos ensaios clínicos foram a ausência de um grupo-controle e a utilização de critérios diagnósticos para a SPM diferentes dos encontrados no DSM-III-R ou no DSM-IV. Os textos na íntegra dos 33 artigos selecionados foram obtidos, e, após sua análise, sete foram excluídos. Portanto 26 referências preencheram todos os critérios de seleção. Todas as 26 foram encontradas no Medline, 25 também na Biblioteca Cochrane, 12 no psycINFO e nenhuma na LILACS. Nenhuma referência que se adequasse aos critérios de inclusão foi encontrada nos artigos obtidos além daquelas selecionadas através das bases de dados. clínicos foram aleatorizados, e apenas um (Pealstein et al., 1997) não foi duplo-cego. Treze deles (Stone et al., 1991; Steiner et al., 1995, 2001, 2003; Su et al., 1997; Pearlstein et al., 1997; Yonkers et al., 1997; Pearlstein et al., 2000; Freeman et al., 2001; Cohen et al., 2002; Miner et al., 2002; Young et al., 1998; Halbreich et al., 2002) tiveram uma etapa prévia de washout com placebo, e seis (Wood et al., 1992; Menkes et al., 1993; Su et al., 1997; Halbreich e Smoller, 1997; Young et al., 1998; Jermain et al., 1999), uma etapa de cruzamento (crossover) (Tabelas 1 e 2).

A clomipramina é um antidepressivo tricíclico que age bloqueando a recaptação de serotonina e de noradrenalina, principalmente da primeira, aumentando assim as atividades

Tabela 1. Uso contínuo de antidepressivos no tratamento da disforia pré-menstrual: ensaios clínicos controlados com placebo

\begin{tabular}{|c|c|c|c|}
\hline & Desenho & Instrumentos & Resultado (eficácia) \\
\hline Sundblad et al. (1992) & CLO $(n=20)$ vs. PLC $(n=20) ; 3$ ciclos; dc, ale & VAS & $\mathrm{CLO}>\mathrm{PLC}$ \\
\hline Stone et al. (1991) & FLU $(n=10)$ vs. PLC $(n=10) ; 2$ ciclos; dc, ale, wsh & DAF & FLU $>$ PLC \\
\hline Wood et al. (1992) & FLU vs. PLC; $n=8 ; 3$ ciclos; dc, ale, crz & COPE & FLU $>$ PLC \\
\hline Menkes et al. (1993) & FLU vs. PLC; $n=16 ; 3$ ciclos; dc, ale, crz & DRF; VAS & FLU $>$ PLC \\
\hline Steiner et al. & FLU $20 \mathrm{mg}$ vs. FLU 60mg vs. PLC; $n=180 ;$ & VAS; PMTS & FLU 20mg = FLU 60mg; \\
\hline$(1995,2001,2003)$ & 6 ciclos; dc, ale, wsh & & FLU 20mg > PLC; FLU 60mg > PLC \\
\hline Özeren et al. (1997) & FLU $(n=18)$ vs. PLC $(n=17) ; 3$ ciclos; dc, ale & COPE & $\mathrm{FLU}>\mathrm{PLC}$ \\
\hline Su et al. (1997) & FLU vs. PLC; $n=17 ; 3$ ciclos; dc, ale, wsh, crz & VAS; DRF & $\mathrm{FLU}>\mathrm{PLC}$ \\
\hline Pearlstein et al. (1997) & FLU $(n=10)$ vs. BUP $(n=12)$ vs. PLC $(n=12)$ & CGI; HAM-D; GAS & CGI: FLU > BUP > PLC \\
\hline $\begin{array}{l}\text { Yonkers et al. (1997), } \\
\text { Pearlstein et al. (2000) }\end{array}$ & $\begin{array}{l}2 \text { ciclos; ale, wsh } \\
\text { SER vs. PLC; } n=200 ; 3 \text { ciclos; dc, ale, wsh }\end{array}$ & $\begin{array}{l}\text { DRSP, SAS, } \\
\text { Q-LES-Q }\end{array}$ & $\begin{array}{l}\text { HAM-D e GAS: FLU > PLC } \\
\text { SER > PLC }\end{array}$ \\
\hline Wikander et al. (1998) & CIT $(n=17)$ vs. PLC $(n=17) ; 3$ ciclos; dc, ale & VAS & $\mathrm{CIT}=\mathrm{PLC}$ \\
\hline Landen et al. (2001) & $\begin{array}{l}\text { BUS }(n=19) \text { vs. NEF }(n=22) \text { vs. PLC }(n=22) \text {; } \\
2 \text { ciclos; dc, ale }\end{array}$ & CGI; VAS & $\begin{array}{l}\text { CGI: NEF = PLC; NEF = BUS; BUS > PLC } \\
\text { VAS: NEF = PLC; BUS } \geq \text { PLC }\end{array}$ \\
\hline Freeman et al. (2001) & VEN vs. PLC; $n=164 ; 4$ ciclos; dc, ale, wsh & DSR & VEN $>$ PLC \\
\hline Eriksson et al. (1995) & $\begin{array}{l}\text { PAR }(n=22) \text { vs. MAP }(n=21) \text { vs. PLC }(n=22) \text {; } \\
3 \text { ciclos; dc, ale }\end{array}$ & VAS & PAR > PLC; PAR > MAP; MAP = PLC \\
\hline Cohen et al. (2004) & PAR[CR] $25 \mathrm{mg}(n=111)$ vs. PAR[CR] $12,5 \mathrm{mg}(n=95)$ & VAS & $\mathrm{PAR}[\mathrm{CR}] 25 \mathrm{mg}=\mathrm{PAR}[\mathrm{CR}] 12,5 \mathrm{mg}>\mathrm{PLC}$ \\
\hline Pearlstein et al. (2005) & $\begin{array}{l}\text { vs. PLC }(n=107) ; 3 \text { ciclos; dc, ale } \\
\text { PAR[CR] } 25 \mathrm{mg}(n=120) \text { vs. PAR[CR] 12,5 mg } \\
(n=115) \text { vs. PLC }(n=124) ; 3 \text { ciclos; dc, ale }\end{array}$ & VAS & $\mathrm{PAR}[\mathrm{CR}] 25 \mathrm{mg}=\mathrm{PAR}[\mathrm{CR}] 12,5 \mathrm{mg}>\mathrm{PLC}$ \\
\hline
\end{tabular}

Fármacos: BUP: bupropiona; BUS: buspirona; CIT: citalopram; CLO: clomipramina; FLU: fluoxetina; MAP: maprotilina; NEF: nefazodona; PAR: paroxetina; PAR[CR] paroxetina de liberação controlada; PLC: placebo; SER: sertralina; VEN: venlafaxina.

Método: dc: duplo-cego; ale: aleatorizado; crz: com cruzamento; wsh: com etapa de washout com placebo.

Escalas: COPE: Calendar of Premenstrual Experiences; GAS: Global Assessment Scale; CGI: Clinical Global Impression; DAF: Daily Assessment Form; DRF: Daily Rating Form; DRSP: Daily Rating of Severity of Problems Form; DSR: Daily Symptom Report; HAM-D: Hamilton Rating Scale for Depression; PMTS: Premenstrual Tension Scale; Q-LES-Q: Quality of Life Enjoyment and Satisfaction Questionnaire; SAS: Social Adjustment Scale; VAS: escala visual analógica.

Vários antidepressivos foram testados em ensaios clínicos controlados no tratamento da DPM. Na maioria dos estudos, a substância foi administrada por todo o ciclo menstrual (uso contínuo), mas em outros, apenas durante a fase lútea, em geral nos 14 dias que precediam o dia previsto para o início da menstruação (uso intermitente). Em todos os ensaios clínicos, a avaliação da eficácia do antidepressivo na DPM era o objetivo primário; e o antidepressivo foi comparado com placebo, sendo que em apenas três deles houve ainda, paralelamente, uma comparação com outra substância ativa: bupropiona (Pearlstein et al., 1997), maprotilina (Eriksson et al.,1995) e buspirona (Landen et al., 2001). Todos os ensaios serotoninérgica e noradrenérgica no SNC. Ela foi o primeiro antidepressivo testado para a DPM em ensaios clínicos controlados. Foram dois estudos: num o medicamento foi empregado de forma contínua (Sundblad et al., 1992), e, no outro, de forma intermitente (Sundblad et al., 1993). Em ambos, a clomipramina, em doses que variaram entre 25 e $75 \mathrm{mg}$ ao dia, foi superior ao placebo.

Todavia os inibidores seletivos da recaptação da serotonina (ISRS), como a fluoxetina, a sertralina, o citalopram e a paroxetina, foram a classe de antidepressivos mais estudada.

A fluoxetina foi comparada com placebo em nove ensaios clínicos: uso contínuo em sete (Menkes et al., 1993; 
Tabela 2. Uso intermitente de antidepressivos no tratamento da disforia pré-menstrual: ensaios clínicos controlados com placebo

\begin{tabular}{llll}
\hline & \multicolumn{1}{c}{ Desenho } & Instrumentos & Resultado (eficácia) \\
\hline Sundblad et al. (1993) & CLO $(n=15)$ vs. PLC $(n=14) ; 3$ ciclos, dc, ale & VAS & CLO > PLC \\
Cohen et al. (2002) & FLU 10mg vs. FLU 20mg vs. PLC; $n=260 ; 3$ ciclos; dc, ale, wsh & DRSP & FLU 20mg > PLC; FLU 10mg = PLC \\
Miner et al. (2002) & FLU 90mg semanal, 1 vez vs. FLU 90mg semanal, 2 vezes vs. & DRSP; PMTS & FLU 2 vezes > FLU 1 vez > PLC \\
& PLC; $n=257 ; 3$ ciclos; dc, ale, wsh & & \\
Halbreich e Smoller (1997) & SER vs. PLC; $n=11 ; 2$ ciclos; dc, ale, crz & DRF & SER > PLC \\
Young et al. (1998) & SER vs. PLC; $n=11 ; 2$ ciclos; dc, ale, crz, wsh & COPE & SER > PLC \\
Jermain et al. (1999) & SER vs. PLC; $n=57 ; 2$ ciclos; dc, ale, crz & COPE & SER > PLC \\
Halbreich et al. (2002) & SER vs. PLC; $n=281 ; 3$ ciclos; dc, ale, wsh & DRSP & SER > PLC \\
Wikander et al. (1998) & CIT $(n=18)$ vs. PLC $(n=17) ; 3$ ciclos; dc, ale & VAS & CIT > PLC \\
Landen et al. (2001) & BUS $(n=19)$ vs. NEF $(n=22)$ vs. PLC $(n=22) ; 2$ ciclos; dc, ale & VAS & NEF $=$ PLC; BUS $=$ PLC \\
Steiner et al. (2005) & PAR[CR] 25mg $(n=116)$ vs. PAR[CR] 12,5mg & VAS & PAR[CR] 25mg = PAR[CR] 12,5mg > PLC \\
& (n=130) vs. PLC $(n=120) ; 2$ ciclos; dc, ale & & \\
\hline
\end{tabular}

Fármacos: BUP: bupropiona; BUS: buspirona; CIT: citalopram; CLO: clomipramina; FLU: fluoxetina; MAP: maprotilina; NEF: nefazodona; PAR: paroxetina; PAR[CR] paroxetina de liberação controlada; PLC: placebo; SER: sertralina; VEN: venlafaxina.

Método: dc: duplo-cego; ale: aleatorizado; crz: com cruzamento; wsh: com etapa de washout com placebo.

Escalas: COPE: Calendar of Premenstrual Experiences; GAS: Global Assessment Scale; CGI: Clinical Global Impression; DAF: Daily Assessment Form; DRF: Daily Rating Form; DRSP: Daily Rating of Severity of Problems Form; DSR: Daily Symptom Report; HAM-D: Hamilton Rating Scale for Depression; PMTS: Premenstrual Tension Scale; Q-LES-Q: Quality of Life Enjoyment and Satisfaction Questionnaire; SAS: Social Adjustment Scale; VAS: escala visual analógica.

Ozeren et al., 1997; Pearlstein et al., 1997; Steiner et al., 1995; Stone et al., 1991; Su et al., 1997; Wood et al., 1992) e uso intermitente em dois (Cohen et al., 2002; Miner et al., 2002). Em todos eles ela foi superior. Na maioria, a dose diária de $20 \mathrm{mg}$ foi a única utilizada. No estudo de Steiner et al. (1995), as doses de $20 \mathrm{mg}$ e $60 \mathrm{mg}$ foram igualmente eficazes, e no de Cohen et al. (2002), a de $10 \mathrm{mg}$ foi inferior à de $20 \mathrm{mg}$. Miner et al. (2002) realizaram o único estudo com a fluoxetina de liberação prolongada (90mg), que é ingerida uma vez a cada sete dias, e observaram maior eficácia quando a substância foi usada nas duas semanas da fase lútea do que quando em apenas uma semana. No estudo de Pearlstein et al. (1997), a fluoxetina foi superior não somente ao placebo, mas também à bupropiona, um antidepressivo que atua nas vias noradrenérgica e dopaminérgica. Observou-se que, além das alterações do humor e do comportamento, também os sintomas físicos (Steiner et al., 2001) e a capacidade para o trabalho (Steiner et al., 2003) poderiam melhorar com a fluoxetina.

Há cinco ensaios clínicos controlados com a sertralina (50-150mg/dia), que em todos eles se mostrou mais eficaz que o placebo. Em quatro deles (Halbreich et al., 2002; Halbreich e Smoller, 1997; Jermain et al.,1999; Young et al., 1998), a substância foi empregada apenas na fase lútea e em um deles (Yonkers et al., 1997), durante todo o ciclo. Nesse mesmo estudo, cujos resultados foram publicados mais tarde por Pearlstein et al. (2000), observou-se efeito benéfico da sertralina também sobre o desempenho psicossocial nas mulheres tratadas.

A paroxetina, por sua vez, foi testada em quatro ensaios clínicos controlados. No estudo de Eriksson et al. (1995), essa substância, usada de forma contínua numa dose de 10 a 20mg/ dia, foi superior ao placebo e à maprotilina, um antidepressivo noradrenérgico. Os outros três ensaios clínicos utilizaram a paroxetina de liberação controlada (paroxetina CR): dois de forma contínua (Cohen et al., 2004; Pearlstein et al., 2005) e um de forma intermitente (Steiner et al., 2005). Nesses três estudos as doses diárias de $12,5 \mathrm{mg}$ e $25 \mathrm{mg}$ se equivaleram, e ambas foram superiores ao placebo.
Apenas Wikander et al. (1998) estudaram o citalopram na DPM. As pacientes que fizeram uso desse antidepressivo durante a fase lútea melhoraram significativamente mais do que aquelas que tomaram placebo; todavia as que fizeram uso do citalopram durante todo o ciclo não se diferenciaram das que tomaram placebo quanto à resposta terapêutica. As doses do citalopram variaram entre 10 e $30 \mathrm{mg}$ ao dia.

Por via de regra, nesses ensaios clínicos os antidepressivos serotoninérgicos foram bem tolerados pelas pacientes.

A nefazodona é um antidepressivo que também bloqueia a recaptação de serotonina, mas sua ação mais importante é como antagonista do receptor serotoninérgico $5-\mathrm{HT}_{2}$. No único ensaio clínico com essa substância na DPM (Landen et al., 2001), a nefazonona, usada tanto de forma contínua como intermitente, não foi melhor do que o placebo ou a buspirona.

O mecanismo de ação da venlafaxina é o bloqueio da recaptação da serotonina, da noradrenalina e, em menor grau, da dopamina. Freeman et al. (2001) observaram que esse antidepressivo, em doses de $50 \mathrm{mg}$ a $200 \mathrm{mg}$ ao dia, foi significativamente mais eficaz que o placebo no tratamento da DPM.

\section{Discussão}

Até o momento, apenas três substâncias foram aprovadas pela Food and Drug Administration (FDA) - órgão do governo norte-americano que regula alimentos e medicamentos - para o tratamento do TDPM: a fluoxetina, a sertralina e a paroxetina CR. Esses medicamentos, todos ISRS, foram os que demonstraram eficácia em um número maior de ensaios clínicos. O citalopram só foi comparado com placebo uma vez, tendo apresentado resultado ambíguo: favorável em uso intermitente, mas não em uso contínuo. Em dois estudos (Eriksson et al., 1995; Pearlstein et al., 1997) os ISRS foram superiores não apenas em relação ao placebo, mas também aos antidepressivos de outras classes, de ação predominantemente noradrenérgica ou dopaminérgica. 
Os efeitos favoráveis da clomipramina e da venlafaxina na DPM também podem estar relacionados a uma ação na transmissão serotoninérgica, pois a clomipramina é um dos mais fortes antagonistas da recaptação de serotonina, e a venlafaxina, quando administrada em doses menores, tem uma ação mais serotoninérgica do que noradrenérgica ou dopaminérgica. $A$ desvantagem da clomipramina, por ser um tricíclico, estaria em sua menor tolerabilidade, quando comparada com os ISRS e outros antidepressivos mais modernos. A nefazodona, que não é um ISRS, parece ser a exceção entre os antidepressivos de ação predominantemente serotoninérgica, pois não se mostrou eficaz no tratamento da DPM.

Alguns ensaios clínicos controlados, mas não todos, apontam boa resposta da SPM ao alprazolam, o qual, porém, como benzodiazepínico, tem grande potencial de causar dependência. Há bons resultados na SPM também com agonistas do hormônio de liberação da gonadotrofina $(\mathrm{GnRH})$, mas esses são menos eficazes nas alterações do humor do que nas físicas, têm o inconveniente de inibir a ovulação e estão associados a risco de cardiotoxicidade e osteoporose. Todavia, nesses estudos não foram utilizados os critérios diagnósticos do DSM-III-R ou do DSM-IV para a DPM (Pearlstein e Steiner, 2000).

Em função dos resultados positivos nos estudos controlados com placebo, o que por enquanto não se observou com outras substâncias, os ISRS são considerados medicamentos de primeira linha no tratamento da DPM. Entretanto faltam comparações diretas entre os antidepressivos e outros tipos de medicamento.

Uma questão que se expõe é a de qual deve ser a duração do tratamento da DPM. Parece razoável mantê-lo por pelo menos um ano, que é o prazo mínimo de avaliação para a formulação do diagnóstico. Diz o bom senso que, uma vez retirado o medicamento, havendo recidiva da sintomatologia, ele deve ser reintroduzido. Em todos os ensaios clínicos estudados, os antidepressivos foram empregados por um período curto, no máximo durante seis ciclos menstruais. Assim, nada é possível dizer quanto a seus resultados num prazo mais longo.

Em nove estudos (Tabela 2), o uso de antidepressivos apenas na fase lútea do ciclo menstrual mostrou-se superior ao de placebo na DPM. Além disso, diversos ensaios clínicos apontam que, na DPM, o uso intermitente de fluoxetina (Steiner et al., 1997), sertralina (Halbreich e Smoller, 1997) e citalopram (Flores-Ramos et al., 2003) não se distingue da utilização contínua da mesma substância quanto à resposta terapêutica. No estudo de Wikander et al. (1998), o citalopram foi mais eficaz quando usado apenas no período pré-menstrual do que

\section{Referências}

Alpay FB, Turhan NO. Intermittent versus continuous sertraline therapy in the treatment of premenstrual dysphoric disorders. Int J Fertil Womens Med, 46: 228-31, 2001.

Cohen LS, Miner C, Brown EW, Freeman E, Halbreich U, Sundell $\mathrm{K}$, Mccray S. Premenstrual daily fluoxetine for premenstrual dysphoric disorder: a placebo-controlled, clinical trial using computerized diaries. Obstet Gynecol, 100: 435-44, 2002. quando durante todo o ciclo. Dessa forma, parece vantajoso, considerando-se a possibilidade de efeitos colaterais e também os custos financeiros para a paciente, restringir a prescrição do antidepressivo aos dias anteriores à menstruação. No entanto Alpay e Turhan (2001), de forma surpreendente, observaram que o uso intermitente da sertralina no tratamento da DPM estava relacionado a efeitos adversos significativamente mais intensos do que o uso contínuo da substância.

Os resultados encontrados nos ensaios clínicos com antidepressivos na DPM não podem ser extrapolados para a SPM, na qual as alterações do humor podem estar ausentes. A experiência clínica mostra que, na SPM, sintomas físicos específicos - não o quadro como um todo - podem responder a tratamentos sintomáticos, como o uso de analgésicos para a cefaléia ou de diuréticos para o edema. Além disso, casos mais leves de SPM não necessariamente precisam ser medicados, poupando assim as pacientes do inevitável risco de efeitos colaterais.

As limitações dessa revisão se referem, primeiramente, à não-utilização de outras bases de dados, como o Embase, por exemplo. Além disso, com relação aos ensaios clínicos, foram raros os que realizaram comparações entre duas ou mais substâncias ativas: a quase totalidade deles utilizou como controle apenas o placebo. Por fim, praticamente todos os estudos foram patrocinados pela indústria farmacêutica, o que pode ter gerado um viés: não sabemos se as publicações representam todas as pesquisas realizadas com antidepressivos na DPM, ou se algumas delas, em que os resultados foram insatisfatórios, jamais foram submetidas a periódicos.

\section{Conclusão}

De acordo com os ensaios clínicos encontrados, os antidepressivos, especialmente os ISRS, parecem ser eficazes na DPM. Todavia restam diversas incertezas quanto à superioridade dessas substâncias sobre psicofármacos ou medicamentos não-psiquiátricos, à duração do tratamento, ao seu efeito a longo prazo e a se o tratamento pode ser feito apenas de forma intermitente.

A boa resposta aos ISRS na DPM pode estar relacionada a uma disfunção serotoninérgica que existiria nas mulheres portadoras desse distúrbio. Há uma relação bastante estreita entre os transtornos do humor e a DPM. Uma hipótese que pode ser levantada é a de que os quadros disfóricos pré-menstruais representam uma seqüela de um episódio depressivo anterior ou, alternativamente, uma forma precursora de um episódio depressivo que mais tarde irá se desenvolver.
Cohen LS, Soares CN, Yonkers KA, Bellew KM, Bridges IM, Steiner $M$. Paroxetine controlled release for premenstrual dysphoric disorder: a double-blind, placebo-controlled trial. Psychosom Med, 66: 707-13, 2004.

Diegoli MSC, Fonseca AM, Diegoli CA, Halbe HW, Bagnoli VR, Pinotti JA. Síndrome pré-menstrual: estudo da incidência e das variações sintomatológicas. Rev Ginecol Obstet, 5: 238-42, 1994. 
Endicott J. History, evolution, and diagnosis of premenstrual dysphoric disorder. J Clin Psychiatry, 61(Suppl 12): 5-8, 2000.

Eriksson E, Hedberg MA, Andersch B, Sundblad C. The serotonin reuptake inhibitor paroxetin is superior to the noradrenaline reuptake inhibitor maprotiline in the treatment of premenstrual syndrome. Neuropsychopharmacology, 12: 167-76, 1995.

Flores-Ramos M, Ontiveros-Uribe M, Cortes-Sotres J. Comparación entre el tratamiento continuo y el intermitente con citalopram para el trastorno disfórico premenstrual. Salud Mental, 26: 37-45, 2003.

Freeman EW, Rickels K, Yonkers KA, Kunz NR, Mcpherson M, Upton $\mathrm{GV}$. Venlafaxine in the treatment of premenstrual dysphoric disorder. Obstet Gynecol, 98: 737-44, 2001.

Halbreich U, Bergeron R, Yonkers KA, Freeman E, Stout AL, Cohen L. Efficacy of intermittent, luteal phase sertraline treatment of premenstrual dysphoric disorder. Obstet Gynecol, 100: 1219-29, 2002.

Halbreich U, Smoller JW. Intermittent luteal phase sertraline treatment of dysphoric premenstrual syndrome. J Clin Psychiatry, 58: 399-402, 1997.

Jermain DM, Preece CK, Sykes RL, Kuehl TJ, Sulak PJ. Luteal phase sertraline treatment for premenstrual dysphoric disorder. Results of a double-blind, placebo-controlled, crossover study. Arch Fam Med, 8: 328-32, 1999.

Landen M, Eriksson O, Sundblad C, Andersch B, Naessen T, Eriksson E. Compounds with affinity for serotonergic receptors in the treatment of premenstrual dysphoria: a comparison of buspirone, nefazodone and placebo. Psychopharmacology (Berl), 155: 292-8, 2001.

Ling FW. Recognizing and treating premenstrual dysphoric disorder in the obstetric, gynecologic, and primary care practices. J Clin Psychiatry, 61(Suppl 12): 9-16, 2000.

Menkes DB, Taghavi E, Mason PA, Howard RC. Fluoxetine's spectrum of action in premenstrual syndrome. Int Clin Psychopharmacol, 8: 95-102, 1993.

Miner C, Brown E, Mccray S, Gonzales J, Wohlreich M. Weekly lutealphase dosing with enteric-coated fluoxetine $90 \mathrm{mg}$ in premenstrual dysphoric disorder: a randomized, double-blind, placebo-controlled clinical trial. Clin Ther, 24: 417-33, 2002.

Nogueira CWM, Silva JLP. Prevalência dos sintomas da síndrome prémenstrual. Rev Bras Ginecol Obstet, 22: 347-51, 2000.

Ozeren S, Corakci A, Yucesoy I, Mercan R, Erhan G. Fluoxetine in the treatment of premenstrual syndrome. Eur J Obstet Gynecol Reprod Biol, 73: 167-70, 1997.

Pearlstein T, Steiner M. Non-antidepressant treatment of premenstrual syndrome. J Clin Psychiatry, 61(Suppl 12): 22-7, 2000.

Pearlstein TB, Bellew KM, Endicott J, Steiner M. Paroxetine controlled release for premenstrual dysphoric disorder: remission analysis following a randomized, double-blind, placebo-controlled trial. Prim Care Companion J Clin Psychiatry, 7: 53-60, 2005.

Pearlstein TB, Halbreich U, Batzar ED, Brown CS, Endicott J, Frank E, Freeman EW, Harrison WM, Haskett RF, Stout AL, Yonkers KA. Psychosocial functioning in women with premenstrual dysphoric disorder before and after treatment with sertraline or placebo. J Clin Psychiatry, 61: 101-9, 2000.

Pearlstein TB, Stone AB, Lund SA, Scheft H, Zlotnick C, Brown WA. Comparison of fluoxetine, bupropion, and placebo in the treatment of premenstrual dysphoric disorder. J Clin Psychopharmacol, 17: 261-6, 1997.

Silva CML, Gigante DP, Carret MLV. Estudo populacional da síndrome pré-menstrual. Rev Saúde Pública, 40: 47-56, 2006.
Steiner M, Brown E, Trzepacz P, Dillon J, Berger C, Carter D, Reid R, Stewart $D$. Fluoxetine improves functional work capacity in women with premenstrual dysphoric disorder. Arch Women Ment Health, 6: 71-7, 2003.

Steiner M, Hirschberg AL, Bergeron R, Holland F, Gee MD, Van Erp E. Luteal-phase dosing with paroxetine controlled release (CR) in the treatment of premenstrual dysphoric disorder. Am J Obstet Gynecol, 193: 352-60, 2005.

Steiner M, Korzekwa M, Lamont J, Wilkins A. Intermittent fluoxetine dosing in the treatment of women with premenstrual dysphoria. Psychopharmacol Bull, 33: 771-4, 1997.

Steiner M, Pearlstein T. Premenstrual dysphoria and the serotonin system: pathophysiology and treatment. J Clin Psychiatry, 61(Suppl 12): 17-21, 2000.

Steiner M, Romano SJ, Babcock S, Dillon J, Shuler C, Berger C, Carter D, Reid R, Stewart D, Steinberg S, Judge R. The efficacy of fluoxetine in improving physical symptoms associated with premenstrual dysphoric disorder. Bjog, 108: 462-8, 2001.

Steiner M, Steinberg S, Stewart D, Carter D, Berger C, Reid R, Grover D, Streiner D. Fluoxetine in the treatment of premenstrual dysphoria. Canadian Fluoxetine/Premenstrual Dysphoria Collaborative Study Group. N Engl J Med, 332: 1529-34, 1995.

Stone AB, Pearlstein TB, Brown WA. Fluoxetine in the treatment of late luteal phase dysphoric disorder. J Clin Psychiatry, 52: 290-3, 1991.

Su TP, Schmidt PJ, Danaceau MA, Tobin MB, Rosenstein DL, Murphy DL, Rubinow DR. Fluoxetine in the treatment of premenstrual dysphoria. Neuropsychopharmacology, 16: 346-56, 1997.

Sundblad C, Hedberg MA, Eriksson E. Clomipramine administered during the luteal phase reduces the symptoms of premenstrual syndrome: a placebo-controlled trial. Neuropsychopharmacology, 9: 133-45, 1993.

Sundblad C, Modigh K, Andersch B, Eriksson E. Clomipramine effectively reduces premenstrual irritability and dysphoria: a placebo-controlled trial. Acta Psychiatr Scand, 85: 39-47, 1992.

Wikander I, Sundblad C, Andersch B, Dagnell I, Zylberstein D, Bengtsson F, Eriksson E. Citalopram in premenstrual dysphoria: is intermittent treatment during luteal phases more effective than continuous medication throughout the menstrual cycle? J Clin Psychopharmacol, 18: 390-8, 1998

Wood SH, Mortola JF, Chan YF, Moossazadeh F, Yen SS. Treatment of premenstrual syndrome with fluoxetine: a double-blind, placebocontrolled, crossover study. Obstet Gynecol, 80: 339-44, 1992.

Wurtman JJ, Brzezinski A, Wurtman RJ, Laferrere B. Effect of nutrient intake on premenstrual depression. Am J Obstet Gynecol, 161: 1228-34, 1989.

Yonkers KA. The association between premenstrual dysphoric disorder and other mood disorders. J Clin Psychiatry, 58(Suppl 15): 19-25, 1997.

Yonkers KA, Halbreich U, Freeman E, Brown C, Endicott J, Frank E, Parry B, Pearlstein T, Severino S, Stout A, Stone A, Harrison W. Symptomatic improvement of premenstrual dysphoric disorder with sertraline treatment. a randomized controlled trial. Sertraline Premenstrual Dysphoric Collaborative Study Group. Jama, 278: 983-8, 1997.

Young SA, Hurt PH, Benedek DM, Howard RS. Treatment of premenstrual dysphoric disorder with sertraline during the luteal phase: a randomized, double-blind, placebo-controlled crossover trial. J Clin Psychiatry, 59: 76-80, 1998. 